\title{
Classificação angiográfica na revascularização do membro inferior isquêmico: pode a angiografia definir a resistência do leito receptor do enxerto?
}

\author{
Angiographic classification in ischemic lower limb revascularization: \\ can it define arterial outflow resistance?
}

\section{Fabio Henrique Rossi, Pedro Puech Leão, Nilo Mitsuro Izukawa, Akash Kuzhiparambil Prakasan*}

\section{Resumo}

Contexto: A arteriografia é muitas vezes utilizada como único método pré-operatório para a definição da conduta terapêutica na revascularização do membro isquêmico, seja ela realizada através de angioplastia transluminal, ou de cirurgia convencional. Ainda hoje, sua utilização é assunto de debate.

Objetivo: Comparar um método de classificação arteriográfica simples com estudos hemodinâmicos pré e intraoperatórios do leito arterial isquêmico a ser revascularizado, com o objetivo de verificar seu poder para definir a resistência desse leito arterial.

Métodos: Foram analisadas 68 cirurgias de revascularização de membros inferiores isquêmicos realizadas no período de julho de 1999 a julho de 2004 no Setor de Cirurgia Vascular do Instituto Dante Pazzanese de Cardiologia. As características do leito arterial receptor do enxerto foram estudadas e comparadas através de método de classificação arteriográfica pré-operatória proposto pelos autores, análise hemodinâmica pré-operatória por eco-Doppler colorido e intraoperatória por medidas diretas de vazão, pressão e resistência.

Resultados: Foram observados índices de correlação de Spearman positivos $(\mathrm{p}<0,05)$ entre o sistema de classificação arteriográfica préoperatória proposto e as medidas hemodinâmicas ultrassonográficas pré-operatórias de volume de fluxo sanguíneo $(\mathrm{p}=0,035)$ e as medidas diretas intraoperatórias de vazão $(\mathrm{p}=0,006)$, pressão $(\mathrm{p}=0,037)$ e resistência $(\mathrm{p}=0,006)$.

Conclusão: O método de classificação arteriográfica pré-operatória proposto pode definir a resistência do leito arterial a ser revascularizado e auxiliar na definição da conduta e do prognóstico da revascularização do membro inferior isquêmico.

Palavras-chave: Angiografia, isquemia, extremidade inferior, ultrassonografia Doppler colorida, resistência.

\section{Introdução}

As revascularizações dos membros inferiores isquêmicos apresentam indicações clínicas e técnicas operatórias já bastante estudadas e definidas ${ }^{1-4}$. Entretanto, metade destas intervenções pode falhar após 1 ano de acompanhamento clínico ${ }^{1,5,6}$.

\begin{abstract}
Background: Angiography is often used as the only preoperative method to define the therapeutic approach for ischemic lower limb revascularization, either by transluminal angioplasty or surgery. Today its use is still controversial.
\end{abstract}

Objective: To compare a simple method of angiographic classification with hemodynamic preoperative and intraoperative studies of the ischemic arterial bed in order to verify its efficacy in defining arterial bed resistance.

Methods: We analyzed 68 cases of surgical revascularization of ischemic lower limbs performed from July 1999 to July 2004 at the Division of Vascular Surgery of Instituto Dante Pazzanese de Cardiologia. The characteristics of the graft recipient arterial runoff were studied and compared using the method of preoperative angiographic classification proposed by the authors, preoperative hemodynamic analysis by color duplex scanning, and intraoperative direct measurements of flow, pressure and resistance.

Results: Positive rates for Spearman correlation $(\mathrm{p}<0.05)$ were observed between the preoperative angiographic classification proposed in this study and the preoperative ultrasound hemodynamic measurements of blood flow volume $(\mathrm{p}=0.035)$ and direct intraoperative measurements of flow $(p=0.006)$, pressure $(p=0.037)$ and resistance $(\mathrm{p}=0.006)$.

Conclusion: The preoperative method of angiographic classification proposed can assess the resistance of the arterial bed to be revascularized and help to define the approach and prognosis of the revascularization of ischemic lower limbs.

Keywords: Angiography, ischemia, lower extremity, ultrasound examination, color Doppler, resistance.

A definição das características do leito distal a ser revascularizado pode nos auxiliar no estabelecimento da melhor estratégia terapêutica e representa uma etapa importantíssima na indicação do tipo de tratamento e da estratégia cirúrgica a ser seguida.

\footnotetext{
* Instituto Dante Pazzanese de Cardiologia, Faculdade de Medicina, Universidade de São Paulo, São Paulo, SP.

Não foram declarados conflitos de interesse associados à publicação deste artigo. Artigo submetido em 23.03.09, aceito em 17.08.09.

J Vasc Bras. 2009;8(3):207-213.

Copyright $\odot 2009$ by Sociedade Brasileira de Angiologia e de Cirurgia Vascular
} 
A revascularização, quando mal indicada, pode levar à obstrução precoce do enxerto e à piora da perfusão do membro, exacerbando a dor, elevando o nível da amputação e dificultando a reabilitação. Além disso, pode pôr em risco a vida do paciente, muitas vezes acometido por comorbidades graves, e elevar os custos médico-hospitalares.

Inúmeros estudos, utilizando os mais diferentes métodos, demonstraram que a resistência ao fluxo sanguíneo do leito isquêmico pode influenciar nos resultados de perviedade e salvamento do membro ${ }^{7-26}$.

A arteriografia pré-operatória, apesar de ser o método mais utilizado e considerado padrão-ouro, pode falhar na definição do prognóstico da revascularização ${ }^{27}$. A Sociedade Norte-Americana de Cirurgia Vascular procurou em duas ocasiões elaborar um sistema de classificação que pudesse melhorar a capacidade da arteriografia no estabelecimento deste prognóstico ${ }^{28,29}$. Apesar disso e da complexidade dos sistemas de classificação arteriográfica propostos, estudos que observaram a sua aplicação clínica demonstraram resultados contraditórios quanto a sua capacidade de estabelecer o prognóstico do enxerto ${ }^{27,30,31}$.

O fluxo sanguíneo arterial pode ser estudado pelo eco-Doppler colorido. No período pré-operatório, o estudo hemodinâmico do fluxo sanguíneo na artéria escolhida para a revascularização do membro inferior isquêmico pode definir a resistência deste leito e o prognóstico do enxerto ${ }^{32-34}$.

No intraoperatório, medidas diretas de vazão, pressão e resistência do leito revascularizado podem definir a resistência e o prognóstico do enxerto ${ }^{10-15}$.

Interessou-nos observar o poder de um sistema de classificação arteriográfica pré-operatório simplificado e intuitivo que considerasse o local escolhido para a realização da anastomose distal, o número de artérias de perna pérvias e as características do arco arterial do pé para definir a resistência deste leito através da comparação com dois métodos hemodinâmicos, um aplicado de forma não invasiva, ainda no período pré-operatório e através do eco-Doppler colorido, e o outro realizado de forma direta no intraoperatório.

\section{Métodos}

Foram analisadas, retrospectivamente, 68 arteriografias obtidas no pré-operatório em cirurgias de revascularização realizadas no período de julho de 1999 a julho de 2004 no Setor de Cirurgia Vascular do Instituto Dante Pazzanese de Cardiologia.
Levando-se em consideração a complexidade da classificação arteriográfica proposta pela Sociedade NorteAmericana de Cirurgia Vascular, foi idealizado um sistema de classificação simples que contemplasse apenas o leito arterial distal ao local escolhido para a realização da anastomose.

Os itens considerados para a obtenção da pontuação na utilização do sistema de classificação arteriográfica proposto foram:

- Sítio da anastomose distal: artéria poplítea supragenicular; artéria poplítea infragenicular; artérias da perna.

- Características arteriográficas de perviedade das artérias da perna: artéria tibial anterior; artéria tibial posterior; artéria fibular.

- Grau de formação do arco pedioso.

Nesta classificação, as maiores pontuações eram obtidas por leitos distais favoráveis para a realização da cirurgia, teoricamente associados a menores resistências ao fluxo sanguíneo. A maior pontuação era obtida quando o local escolhido para a realização da anastomose distal era a artéria poplítea supragenicular, com as três artérias da perna pérvias e o arco pedioso completo.

Nas artérias da perna, a pontuação máxima (4) era obtida quando a artéria distal ao local da anastomose se encontrava sem obstrução e em confluência com um arco pedioso completo (Tabela 1).

Ainda no período pré-operatório, os pacientes foram submetidos ao estudo eco-Doppler colorido. No local escolhido para a realização da anastomose distal, realizava-se a mensuração do volume de fluxo sanguíneo. Para o cálculo desse volume de fluxo, o maior diâmetro interno da artéria (média de três mensurações realizadas no mesmo local) era medido em corte transversal (modo-B) e o espectro do Doppler pulsado era obtido em corte longitudinal no mesmo local em que fora medido o diâmetro. $\mathrm{O}$ ângulo do Doppler era corrigido, alinhado ao fluxo sanguíneo até $60^{\circ}$ (considerando-se o eixo longitudinal do vaso estudado), e a velocidade integral da curva de velocidades do Doppler da amostra, calculada: volume $(\mathrm{mL} / \mathrm{min})=$ área $\left(\pi r^{2}\right) \times$ velocidade $(\mathrm{cm} / \mathrm{s}) \times$ frequência cardíaca ${ }^{34,35}$.

Após a escolha do leito arterial a ser revascularizado, definido através dos métodos descritos acima, os pacientes foram submetidos à cirurgia. O substituto arterial usado foi 
Tabela 1 - Classificação arteriográfica pré-operatória proposta do leito receptor do enxerto

\begin{tabular}{|c|c|c|c|}
\hline \multicolumn{4}{|c|}{ I - Local escolhido para anastomose distal } \\
\hline Pontuação & 0 & 1 & 2 \\
\hline & Artérias da perna & Artéria poplítea infragenicular & Artéria poplítea supragenicular \\
\hline \multicolumn{4}{|c|}{ II - Características arteriográficas das artérias da perna e arco pedioso } \\
\hline Pontuação & 0 & 1 & 2 \\
\hline Artéria tibial anterior & Oclusão & $\begin{array}{c}\text { - Estenose }>50 \% \\
\text { - Ateromatose difusa }\end{array}$ & Pérvia \\
\hline Artéria tibial posterior & Oclusão & $\begin{array}{c}\text { - Estenose }>50 \% \\
\text { - Ateromatose difusa }\end{array}$ & Pérvia \\
\hline Artéria fibular & Oclusão & $\begin{array}{c}\text { - Estenose }>50 \% \\
\text { - Ateromatose difusa }\end{array}$ & Pérvia \\
\hline Arco pedioso & Ausente & - Incompleto & Pérvio \\
\hline
\end{tabular}

Pontuação total (I + II), índice (máximo/mínimo):

- artéria poplítea supragenicular: 10 (máximo, todas as artérias pérvias) / 2 (mínimo, seguimento isolado de poplítea);

- artéria poplítea infragenicular: 9 (máximo) / 1 (mínimo);

- artérias da perna: 4 (máximo) / 1 (mínimo).

o enxerto venoso reverso em $42(61,8 \%)$ casos, in situ em seis $(8,8 \%)$ casos e translocado em um $(1,5 \%)$ caso, sendo a prótese (PTFE $6 \mathrm{~mm}$ ) empregada em 19 (27,9\%) casos. A anastomose distal do enxerto arterial foi realizada na artéria poplítea supragenicular em $20(29,4 \%)$ casos, na infragenicular em nove $(13,2 \%)$ casos, na artéria tibial anterior em $14(20,6 \%)$ casos, na artéria fibular em $16(23,5 \%)$ casos, na artéria tibial posterior em oito $(11,8 \%)$ casos e no tronco tibiofibular em um $(1,5 \%)$ caso.

No intraoperatório, após a exposição da artéria selecionada para realização da anastomose distal, introduzia-se a sonda uretral em direção caudal e, em seguida, uma bolsa de soro fisiológico era posicionada a $120 \mathrm{~cm}$ de altura em relação à arteriotomia e conectada com a sonda uretral, tendo como intermediário um equipamento de medição de pressão venosa central (PVC). Após a abertura do sistema, mensurava-se a vazão de soro fisiológico em 1 minuto. Estando o equipo de PVC já repleto com solução fisiológica alinhada junto à régua na altura de $120 \mathrm{~cm}$, procedia-se à abertura do equipo e observava-se, durante aproximadamente 1 minuto, a coluna de soro fisiológico reduzir gradativamente em altura até estacionar no ponto de equilíbrio com a pressão da artéria cateterizada. Considerando-se que as variáveis pressão e vazão tendem a interferir de maneira inversa no funcionamento do enxerto, o índice de resistência também foi observado ${ }^{15}$ (Figura 1).

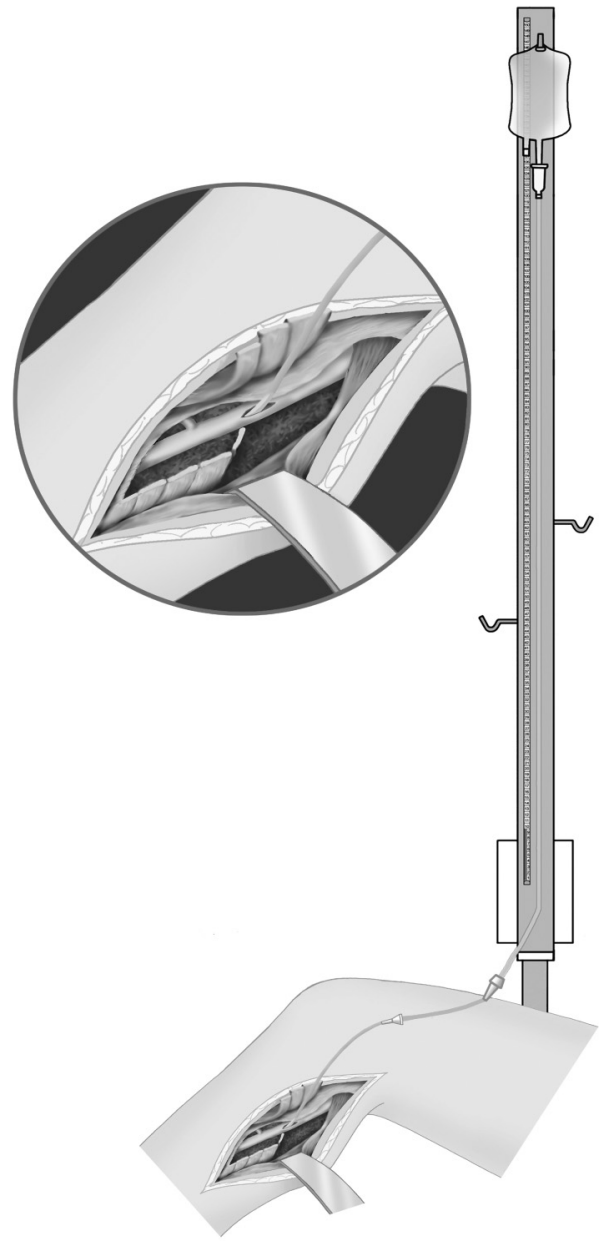

Figura 1 - Sistema de medição de vazão, pressão e resistência intraoperatória 
Para a análise do potencial do sistema de classificação arteriográfica proposto para definir as características hemodinâmicas do leito arterial a ser revascularizado e o prognóstico do enxerto foram realizados estudos do grau de correlação deste método com os métodos hemodinâmicos pré e intraoperatórios descritos acima. Utilizou-se para isso a correlação proposta por Spearman, pois se verificou curva de distribuição não normal no sistema de classificação arteriográfica proposta.

\section{Resultados}

Observou-se que os maiores índices de classificação arteriográfica obtidos coincidiram com leitos distais em que havia maior volume de fluxo sanguíneo ao eco-Doppler pré-operatório (Figura 2).

O mesmo aconteceu em relação às medidas de vazão (Figura 3), pressão (Figura 4) e resistência (Figura 5) do leito distal obtidas no intraoperatório.

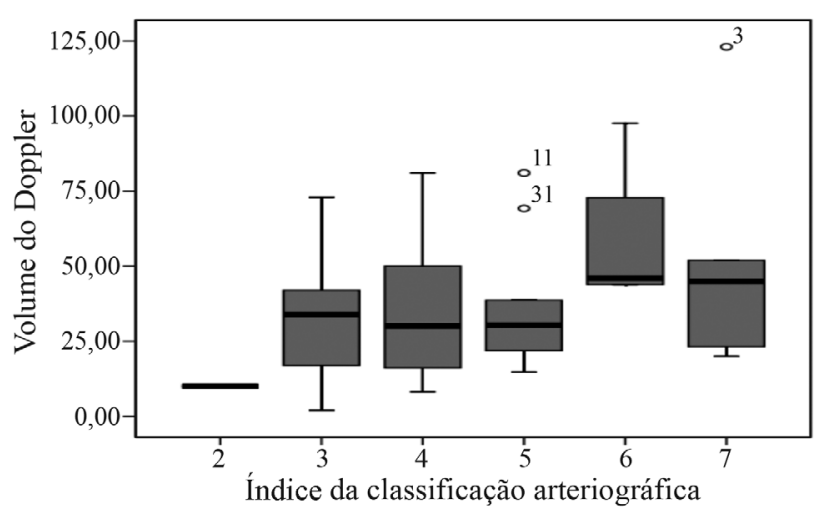

Figura 2 - Comparação entre índice de classificação arteriográfica e volume de fluxo sanguíneo $(\mathrm{mL} / \mathrm{s})$ ao eco-Doppler pré-operatório

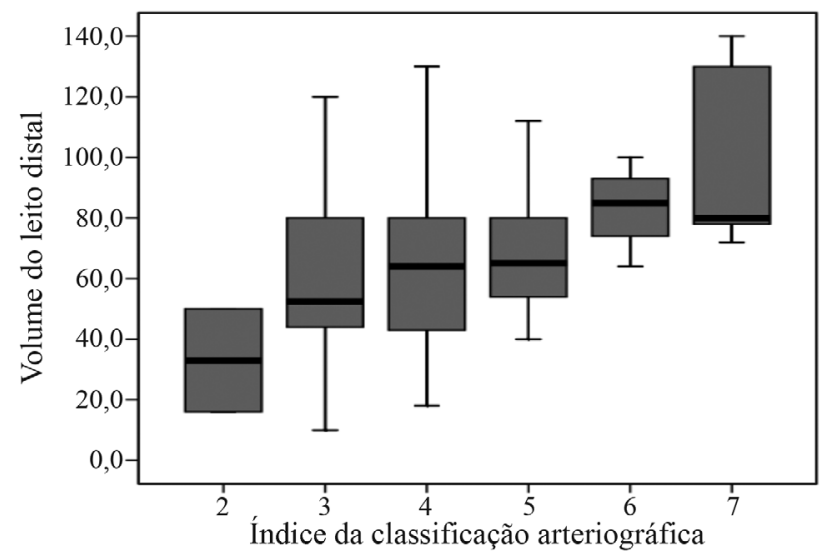

Figura 3 - Comparação entre índice de classificação arteriográfica e vazão $(\mathrm{mL} / \mathrm{min})$ do leito distal no intraoperatório
Observou-se a existência de uma correlação positiva entre a classificação arteriográfica e as medidas de pressão, volume e resistência intraoperatória e o volume de fluxo sanguíneo pré-operatório obtido através do eco-Doppler colorido (Tabela 2).

\section{Discussão}

A perviedade do enxerto na cirurgia de revascularização arterial do membro isquêmico pode ser afetada por uma série de fatores. A resistência do leito arterial receptor do enxerto pode ser causa de falência precoce do mesmo ${ }^{1-6}$.

Tradicionalmente, a arteriografia pré-operatória é o método mais utilizado na definição da conduta terapêutica e no estabelecimento da estratégia de revascularização do membro isquêmico. Entretanto, uma série de circunstâncias pode levar a falha do método em definir as característi-

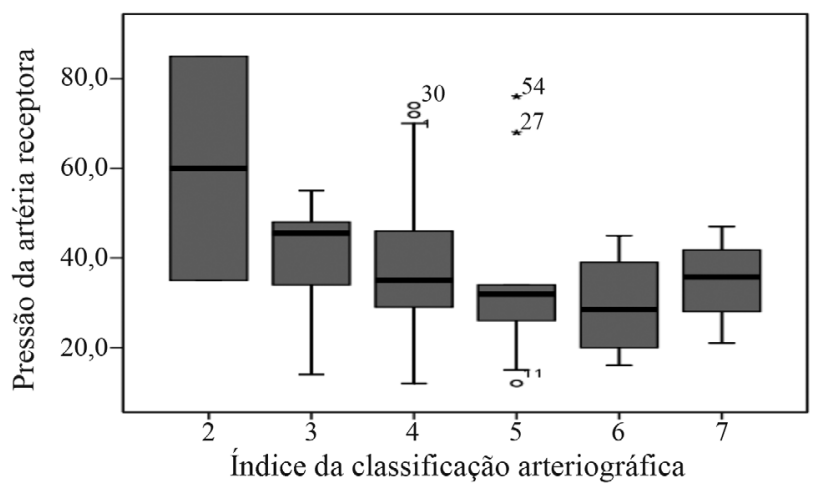

Figura 4 - Comparação entre índice de classificação arteriográfica e pressão ( $\mathrm{cm}$ de soro fisiológico) do leito distal no intraoperatório

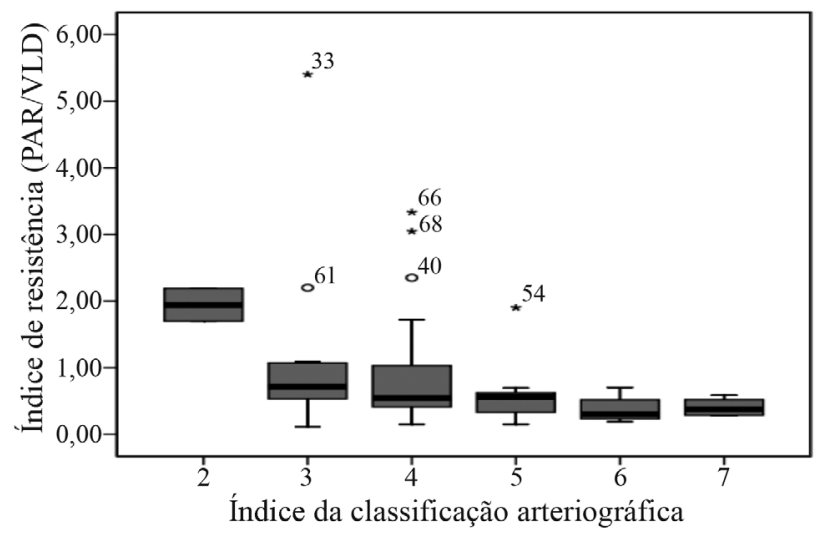

$\mathrm{PAR}=$ pressão da artéria receptora; $\mathrm{VLD}=$ vazão do leito distal.

Figura 5 - Comparação entre índice de classificação arteriográfica e resistência (pressão/vazão) do leito distal no intraoperatório 
Tabela 2 - Índice de correlação de Spearman entre a classificação arteriográfica (IDPC) proposta e medidas de volume de fluxo ultrassonográficas pré-operatórias e medidas de pressão, vazão e resistência intraoperatórias

\begin{tabular}{lcccccc}
\hline & \multicolumn{2}{c}{ Eco-Doppler pré-operatório } & & \multicolumn{3}{c}{ Medidas intraoperatórias } \\
\cline { 2 - 2 } & Volume & & Vazão & Pressão & Resistência \\
\hline Classificação arteriográfica, ICS, valor de $p$ & 0,$257 ; 0,035$ & & 0,$328 ; 0,006$ & $-0,256 ; 0,037$ & $-0,336 ; 0,006$ \\
\hline
\end{tabular}

ICS = índice de correlação de Spearman.

cas do leito arterial distal. Para que haja a presença do meio de contraste no leito arterial estudado, este deve vencer a mesma resistência ao fluxo que tornara a perfusão sanguínea do membro isquêmico insuficiente. Além disso, técnicas de diluição, velocidade e volume de infusão e definição de parâmetros radiográficos podem influenciar nos resultados obtidos. O baixo débito cardíaco, muitas vezes presente no paciente portador de aterosclerose grave e difusa, pode diminuir sobremaneira o fluxo sanguíneo. A presença de múltiplas regiões de obstrução arterial, o aumento da viscosidade sanguínea, o baixo desenvolvimento de sistema de colaterais e a desidratação são outros fatores que influenciam negativamente na chegada do fluxo sanguíneo e do meio de contraste ao segmento isquêmico do membro ${ }^{18}$.

Estes fatores podem ter influenciado os resultados conflitantes obtidos na análise do poder da arteriografia para definir o prognóstico do enxerto na cirurgia de revascularização do membro isquêmico.

Uma série de novos conceitos foram obtidos através da análise não invasiva do fluxo sanguíneo no membro inferior isquêmico. Em estudo realizado em 2006 para obtenção de tese de doutoramento pelo autor principal deste artigo foi demonstrado que o eco-Doppler pré-operatório poderia definir as características hemodinâmicas do leito arterial revascularizado e o prognóstico do enxerto. Foram comparadas medidas de volume de fluxo, obtidas com o ultrassom na artéria escolhida para a realização do enxerto, com medidas diretas de vazão, pressão e resistência obtidas no intraoperatório na mesma artéria, mediante um método reconhecidamente capaz de prever a perviedade do enxerto, e ficou demonstrado haver uma correlação positi$\mathrm{va}^{34,35}$.

No entanto, apesar da existência dessa correlação positiva, alguns fatores devem ser enfatizados. A estimativa do volume de fluxo sanguíneo pelo Doppler pré-operatório apresenta um importante fator técnico limitante. A mensu- ração precisa do diâmetro arterial pode ser difícil, principalmente quando esta é realizada nas artérias da perna. Uma pequena variação da medida do diâmetro nestas pequenas artérias pode representar uma modificação considerável no volume de fluxo. Em relação às medidas diretas intraoperatórias, devemos lembrar que, por se tratar de método invasivo, fatores como espasmo arterial, posição do paciente e características da parede arterial podem influenciar nos resultados das medidas obtidas.

As arteriografias pré-operatórias realizadas no estudo foram classificadas de acordo com as características do leito arterial distal ao sítio escolhido para a realização da anastomose distal do enxerto através do método descrito acima. Esse sistema revelou-se simples, intuitivo e de fácil execução, uma vez que apenas o leito receptor do enxerto é analisado.

Apesar do pequeno número de casos estudados, parece haver uma relação de proporcionalidade entre a pontuação obtida pela classificação arteriográfica proposta e o volume de fluxo obtido ao eco-Doppler e as medidas diretas de vazão, pressão e resistência intraoperatórias observadas nas figuras expostas acima. Parece haver, também, correlação positiva entre a pontuação arteriográfica proposta e as medidas hemodinâmicas pré e intraoperatórias obtidas por métodos previamente estudados e que demonstraram poder estabelecer a resistência e o prognóstico do enxerto $^{15,34,35}$.

\section{Conclusão}

A arteriografia pré-operatória, através do sistema de classificação arteriográfica proposto neste estudo, pode auxiliar na definição das características do leito arterial receptor do enxerto no membro inferior isquêmico e, possivelmente, no estabelecimento do prognóstico do mesmo enxerto. Dessa forma, propõe-se a realização de estudos multicêntricos com seguimento a longo prazo para a verificação da aplicabilidade clínica desta nova classificação arteriográfica. 


\section{Referências}

1. O'Donnell JA, Brener BJ, Brief DK, Alpert J, Parsonnet V. Realistic expectation for patients having lower extremity bypass surgery for limb salvage. Arch Surg. 1977;112:1356-63.

2. Veith FJ, Gupta SK, Ascer E, et al. Six-year prospective multicenter randomized comparison of autologous saphenous vein and expanded polytetrafluoroethylene grafts in infrainguinal arterial reconstructions. J Vasc Surg. 1986;3:104-14.

3. Pomposelli Jr FB, Jepsen SJ, Gibbons GW, et al. Efficacy of the dorsal pedal bypass for limb salvage in diabetic patients: short-term observations. J Vasc Surg. 1990;11:745-51.

4. Bell PR. Femoro-distal grafts - can the results be improved? Eur J Vasc Surg. 1991;5:607-9.

5. Bergamini TM, Towne JB, Bandyk DF, Seabrook GR, Schmitt DD. Experience with in situ saphenous vein bypasses during 1981 to 1989: determinant factors of long-term patency. J Vasc Surg. 1991;13:137-47.

6. Second European Consensus Document on chronic critical leg ischemia. Eur J Vasc Surg. 1992;6 Suppl A:1-32.

7. Bernhard VM. Intraoperative monitoring of femorotibial bypass grafts. Surg Clin North Am. 1974;54:77-84.

8. Dardik H, Ibrahim IM, Sussman B, et al. Morphologic structure of the pedal arch and its relationship to patency of crural vascular reconstruction. Surg Gynecol Obstet. 1981;152:645-8.

9. Liebman PR, Menzoian JO, Mannick JA, Lowney BW, LoGerfo FW. Intraoperative arteriography in femoropopliteal and femorotibial bypass grafts. Arch Surg. 1981;116:1019-21.

10. Ascer E, Veith FJ, Morin L, et al. Quantitative assessment of outflow resistance in lower extremity arterial reconstructions. J Surg Res. 1984;37:8-15.

11. Parvin SD, Evans DH, Bell PR. Peripheral resistance measurement in the assessment of severe peripheral vascular disease. Br J Surg. 1985;72:751-3.

12. Bandyk DF, Cato RF, Towne JB. A low flow velocity predicts failure of femoropopliteal and femorotibial bypass grafts. Surgery. 1985;98:799-809.

13. Stirnemann P, Triller J. The fate of femoropopliteal and femorodistal bypass grafts in relation to intraoperative flow measurement: an analysis of 100 consecutive reconstructions for limb salvage. Surgery. 1986;100:38-44.

14. Ascer E, Veith FJ, White-Flores SA, Morin L, Gupta SK, Lesser ML. Intraoperative outflow resistance as a predictor of late patency of femoropopliteal and infrapopliteal arterial bypasses. J Vasc Surg. 1987;5:820-7.

15. Oliveira LA, Puech-Leão P. Avaliação do leito vascular receptor de enxertos arteriais pela perfusão com pressão constante. J Vasc Bras. 1988;14:120-34.

16. Peterkin GA, Manabe S, LaMorte WW, Menzoian JO. Evaluation of a proposed standard reporting system for preoperative angiograms in infrainguinal bypass procedures: angiographic correlates of measured runoff resistance. J Vasc Surg. 1988;7:379-85.

17. Scott DJ, Vowden P, Beard JD, Horrocks M. Non-invasive estimation of peripheral resistance using pulse generated runoff before femorodistal bypass. Br J Surg. 1990;77:391-5.
18. Okadome K, Onohara T, Yamamura S, Mii S, Sugimachi K. Evaluation of proposed standards for runoff in femoropopliteal arterial reconstructions: correlation between runoff score and flow waveform pattern. A preliminary report. J Cardiovasc Surg (Torino). 1991;32:353-9.

19. Bagi P, Sillesen H, Bitsch K, Hansen HJ. Doppler waveform analysis in evaluation of occlusive arterial disease in the lower limb: comparison with distal blood pressure measurement and arteriography. Eur J Vasc Surg. 1990;4:305-11.

20. Cooper GG, Austin C, Fitzsimmons E, Brannigan PD, Hood JM, D'Sa AA. Outflow resistance and early occlusion of infrainguinal bypass grafts. Eur J Vasc Surg. 1990;4:279-83.

21. Davies AH, Magee TR, Parry R, et al. Duplex ultrasonography and pulse-generated run-off in selecting claudicants for femoropopliteal angioplasty. Br J Surg. 1992;79:894-6.

22. Thompson MM, Sayers RD, Beard JD, Hartshorne T, Brennan JA, Bell PR. The role of pulse-generated run-off, doppler ultrasound and conventional arteriography in the assessment of patients prior to femorocrural bypass grafting. Eur J Vasc Surg. 1993;7:37-40.

23. O'Brien TS, Thomas H, Crow A, Lamont PM. Calf vessel preservation in peripheral vascular disease-angiography versus pulse generated run-off. Eur J Vasc Surg. 1993;7:177-9.

24. Thompson-Fawcett M, Moon M, Hands L, Collin J. The significance of donor leg distal runoff in femorofemoral bypass grafting. Aust N Z J Surg. 1998;68:493-7.

25. Alback A, Biancari F, Saarinen O, Lepantalo M. Prediction of the immediate outcome of femoropopliteal saphenous vein bypass by angiographic runoff score. Eur J Vasc Endovasc Surg. 1998;15:220-4.

26. Alback A, Roth WD, Ihlberg L, Biancari F, Lepantalo M. Preoperative angiographic score and intraoperative flow as predictors of the mid-term patency of infrapopliteal bypass grafts. Eur J Vasc Endovasc Surg. 2000;20:447-53.

27. Takolander R, Fischer-Colbrie W, Jogestrand T, Ohlsen H, Olofsson P, Swedenborg J. The "ad hoc" estimation of outflow does not predict patency of infrainguinal reconstructions. Eur J Vasc Endovasc Surg. 1995;10:187-91.

28. Prepared by the Ad Hoc Committee on Reporting Standards, Society for Vascular Surgery/North American Chapter, International Society for Cardiovascular Surgery. J Vasc Surg. 1986;4:80-94.

29. Rutherford RB, Baker JD, Ernst C, et al. Recommended standards for reports dealing with lower extremity ischemia: revised version. J Vasc Surg. 1997;26:517-38.

30. Liebman PR, Menzoian JO, Mannick JA, Lowney BW, LoGerfo FW. Intraoperative arteriography in femoropopliteal and femorotibial bypass grafts. Arch Surg. 1981;116:1019-21.

31. Rutherford RB, Jones DN, Bergentz SE, et al. Factors affecting the patency of infrainguinal bypass. J Vasc Surg. 1988;8:236-46.

32. Wilson N, Goldberg SJ, Dickinson DF, Scott O. Normal intracardiac and great artery blood velocity measurements by pulsed doppler echocardiography. $\mathrm{Br}$ Heart J. 1985;53:451-8.

33. Lewis P, Psaila JV, Davies WT, McCarty K, Woodcock JP. Measurement of volume flow in the human common femoral 
artery using a duplex ultrasound system. Ultrasound Med Biol. 1986;12:777-84.

34. Henrique Rossi F, Puech-Leão P, Mitsuro Izukawa N, et al. Color-flow duplex hemodynamic assessment of runoff in ischemic lower limb revascularization. Vascular. 2006;14:149-55.

35. Rossi FH. Avaliação do leito arterial distal em revascularização de membros inferiores: estudo comparativo entre ecografia-doppler, arteriografia e medidas diretas de fluxo no intra-operatório [tese]. São Paulo: Universidade de São Paulo; 2006 .

Correspondência:

Fabio Henrique Rossi

Al Jurupis, 900, 103/Iv

CEP 04088-002 - Moema, SP

E-mail: vascular369@hotmail.com 